\title{
An Edge-Based Macao License Plate Recognition System
}

\author{
Chi-Man Pun*, Wai-Yiu Ho \\ Department of Computer and Information Science, University of Macau \\ Macau SAR, China \\ E-mail: \{cmpun,ma46511\}@umac.mo
}

Received: 19-05-2010

Accepted: 31-01-2010

\begin{abstract}
This paper presents a system to recognize Macao license plates. Sobel edge detector is employed to extract the vertical edges, and an edge composition algorithm is proposed to combine the edges into candidate plate regions. They are further examined on the existence of the character " $\mathrm{M}$ " by a verification algorithm. A row separation algorithm is also proposed to cater both one-row and two-row types of plates. Projection analysis and template matching methods are exploited to segment and recognize the characters. Various pre and post processing steps are proposed other than traditional implementation so as to improve the recognition accuracy. This work achieves a high recognition rate of $95 \%$.
\end{abstract}

Keywords: License plate recognition, edge detection, projection, morphology, template matching.

\section{Introduction}

In Macao, automobile is one of the most important transportation methods. As vehicles get more and more, many problems arise such as traffic monitoring, parking lots management, security control of restricted areas, traffic law enforcement, automatic toll collection, etc. The traditional solutions are employing labor to manage these situations, which requires intensive manpower, time and money. During the past decades, Intelligent Transport System technology has been increasingly developed in order to provide better solutions for the above issues. One of the most important topics is the License Plate Recognition (LPR).

In general, LPR consists of three stages. The first stage is locating the license plate from the input image, namely license plate extraction. The second stage is separating the plate into segments according to the alphabets and numerals on it. The last stage is character recognition to identify the segments into machine editable data for other application's use. There have been many research works with algorithms proposed that are able to resolve these problems in various countries, however LPR solutions are usually country specific, and challenges still exist that yet to be tackled which can fall into two categories: license plate variations and environmental variations.

In Macao, license plate has a standard [14] as required by the government, however, the standard itself allows various plate formats and the enforcement is not restrictive. Typically Macao license plate is black in background color and white or yellow in characters. It consists of two components: alphabet of 1 or 2 characters, and numeral of 4 characters. According to number of rows, it can be further divided into two types:

${ }^{*}$ Corresponding author. 
one-row and two-row. For one-row type, the standard size is 520 millimeters height and 120 millimeters width, with the alphabet and numeral components separated by a hyphen. For two-row type, the standard size is 340 millimeters height and 230 millimeters width, with the alphabet component is placed in the first row while the numeral component is placed in the second row. Furthermore, some of the plates have red background and white characters that are for temporary registration of new cars and they will eventually be replaced with regular ones. This is illustrated in Figure 1.

\begin{tabular}{|c|c|}
\hline Regular Plate (One-Row) & ME-60-78 \\
\hline Regular Plate (Tow-Row) & MK \\
& $49-47$ \\
\hline Temporary Plate & EX 620 \\
\hline
\end{tabular}

Figure 1: Different types of Macao license plates

With this specification, the issues of the two types of variations can be summarized as follows:

(1) License plate variations

Size - This is due to the two types of license plates (one-row vs. two-row), and also the position between the camera and the vehicle will affect the size of the plate appears in the input image.

Character spacing - Usually, characters of Macao license plates have an aspect ratio (width-to-height) of 5:7 and a fixed spacing of 10 millimeters between each character. Older vehicles employ old character fonts whose font size is bigger than the current ones and thus less space between characters. An example is given in Figure 2(a). At the same time, through observation we notice that for characters "I" and " 1 ", there is lack of consistence in character spacing. Examples are given in Figure 2(b).

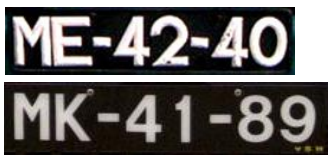

(a)



(b)
Figure 2: Example of variations in character spacing

Multiple license plates - Macao is geographically bordering to China, and China Government allows vehicles from Macao to travel in China with registered China license plates shown. Thus, some vehicles have two license plates shown in their front end and rear end.
This is confusing the exaction algorithms. An example is given in Figure 3.

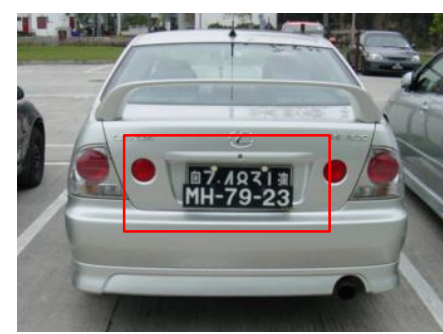

Figure 3: Example of multiple license plates

Location and Orientation - Plates may exist in different locations of an image depends on the physical location of a plate on the vehicle and the camera position. Orientation issue happens due to similar issues, which causes the plate slanted in the input image.

\section{(2) Environmental variations}

Illumination - Different types of illumination may occur in input images, due to environmental lighting (daytime, nighttime, different weather conditions). This degrades the captured image such as low contrast and discoloration, and makes the plate extraction difficult.

Plate-like background patterns - An input image may contain background with pattern similar to plates, such as characters, logos stamped on vehicle and bumpers. This always causes LPR algorithms mis-recognize the location of plates.

Many researchers have been working on solutions for the problems of LPR. For license plate extraction, it could be implemented by two categories of approaches: (i) edge-based methods $[1,2,3,4]$; (ii) color-based methods such as fuzzy logic [5] and neural network [6]. For character segmentation, several techniques have been developed such as projection $[7,8]$ and connected component analysis [9,10]. For character recognition, several algorithms have also been used, such as template matching [11] and neural networks [12]. Among these, two literatures focused on solving Macao LPR problems. Wu et al. [4] proposed a LPR system by exploiting morphological operations to locate the plate region with projection and template matching to recognize the characters, which achieved an extraction rate of $93.2 \%$ and an overall recognition rate of $82.3 \%$. In [13], the authors followed the approach in [2] and implemented a license plate extraction scheme with vertical edge analysis. It achieved an extraction rate of $95 \%$, however the system was incomplete that corresponding character segmentation and recognition solution was not available. Moreover, both methods did 
not accommodate the concern on license plate and environmental variations. Base on the work of [13], we have enhanced the extraction algorithm and proposed a complete system on Macao LPR which is able to deal with the captioned situations as well as improvement in accuracy.

The rest of this paper is organized as follows. In Section 2, the major steps of license plate extraction algorithm are presented. The details of character segmentation and recognition algorithms are described in Section 3 and 4 respectively. Finally, experimental results and comparison to existing works are provided in Section 5, and Section 6 concludes the paper.

\section{License Plate Extraction}

The process flow of our extraction mechanism is given in Figure 4.

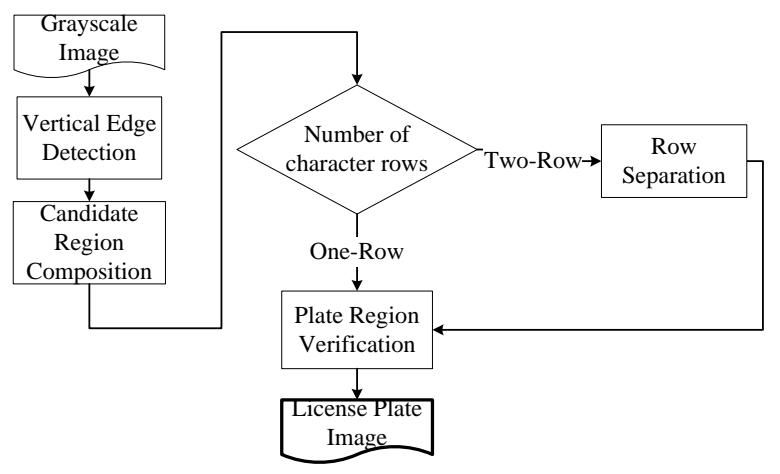

Figure 4: Process flow of license plate extraction

In the Vertical Edge Detection step, vertical edges in the original image are detected by the Sobel edge detection algorithm. The next step is the Candidate Region Composition in which the edge points are merged into lines and then rectangles. They are considered as candidate regions that a license plate may exist. Finally in the Plate Region Verification step, various features of the candidate regions are examined and one of them is selected as the ultimate plate region and being further processed in the character segmentation phase. The mechanism is straightforward for one-row plates and one more step is needed for tworow plates, namely Row Separation.

\subsection{Vertical Edge Detection}

The goal of edge detection is to identify the boundaries of license plate and character objects in the input image. For Macao plates, the contrast of background and character color gives high gradient property, and it is noticed that most vehicles have more horizontal lines (e.g. radiators and bumpers) than vertical lines. Thus vertical edge is employed to detect the license plate.

The input image is first pre-processed by histogram equalization to reduce the illumination effect. The Gradient method is the most popular method to compute digital gradient of an image, which is accomplished by convolving a $3 \times 3$ Sobel operator to each pixel $(i, j)$ as shown in Eq. (1), where $f$ is the image:

$$
G_{x}=\left[\begin{array}{lll}
-1 & 0 & 1 \\
-2 & 0 & 2 \\
-1 & 0 & 1
\end{array}\right] * f
$$

A threshold is applied to $G_{x}$ to suppress the pixels with weaker intensity into background. We name this resulting signal as the vertical edge map $e$ thereafter in this paper.

\subsection{Candidate Region Composition}

In the candidate region composition step, the vertical edges are combined into rectangles to highlight the regions with high vertical gradient. They are evaluated by several features that invalid ones are filtered, and the remaining ones are considered as candidate regions.

In the vertical edge map, edge points in each row is scanned in horizontal direction and compared with the others. If the horizontal distance between the current point and the previous one is less than a threshold ${ }_{t h_{P T} \text { _DIST }}$, the two points are combined into a line. This process continues until a point exceeds the threshold and it becomes the starting point of another line. This way, a group of lines are formed. The criterion to choose ${ }_{t h_{P T} D I S T}$ is to allow the combination process to connect as much edge points of the characters as possible. We already know that a Macao license plate contains eight characters at maximum. Assume that the width of any license plate object will not exceed oneforth of the image width, the maximum possible distance between two edge points will be two characters' width (consider the scenario that there is a hyphen between characters like "A", " 1 ", or " 4 "). This way, we select ${ }^{t h_{P T_{-}} D I S T}$ as Eq. (2).

$$
t h_{P T_{-} D I S T}=w_{I M G} \times \frac{1}{4} \times \frac{1}{8} \times 2=0.0625 \times w_{I M G}
$$

where ${ }^{w_{I M G}}$ is the width of the input image, and 8 is an estimated ratio of width of character to license plate. For a line inside the plate region, the ratio between its 
length and the number of combined edge points should be within a range in relation to the number of character on the Macao license plate. Lines with the ratio out of the range $\left[r_{P T_{-} D I S T_{\text {min }}}, r_{P T_{-} D I S T_{\text {max }}}\right]$ are suppressed to the background. This ratio reaches its minimum value when the actual length of the line is almost the same as the number of combined points (high density), while it reaches its maximum value when two points manage to reach the threshold ${ }_{t h_{P T}{ }_{D} I S T}$ and combined (low density). Thus, we select $\left[r_{P T_{-} D I S T_{\text {min }}}, r_{P T_{-} D I S T \text { max }}\right]$ as Eq. (3) and (4) respectively.

$$
\begin{gathered}
r_{P T_{-} D I S T \text { min }}=1 \\
r_{P T_{-} \text {DIST max }}=\frac{t h_{P T_{-} D I S T}}{2}
\end{gathered}
$$

The lines are then combined into rectangles with a process similar to the process in the last section. The lines in each row are scanned in vertical direction from bottom to up. Each of them is either contributing to a new rectangle, or being merged into an existing rectangle. For the latter case, it must fulfill two criteria as below:

(1) The vertical distance between the current line and the previous line is less than a threshold ${ }_{t h_{L N} D I S T}$. This threshold is set as the distance of spacing between the two character rows in two-row license plates (In one-row type, the lines are closed to each other) as given in Eq. (5).

$$
t h_{L N_{-} D I S T}=w_{I M G} \times \frac{1}{4} \times 0.67 \times \frac{1}{15}=0.01 \times w_{I M G}
$$

In Eq. (5), 0.67 is the aspect ratio of the two-row license plate and 15 is an estimated ratio of height of the space between the two rows to license plate.

(2) The current line overlaps with the rectangle's initial contributing line in the horizontal direction. This is illustrated in Figure 5. In the cases of partial overlap and outer overlap, the excess portion forms another line and being scanned later. If the current line does not fit the above criteria, it will contribute to the next rectangle. Furthermore, the width of a rectangle is determined by the width of the initial contributing line, while the height is the vertical distance between the initial and the last contributing lines.



Figure 5: Cases of overlapping lines
The last step of the composition process is merging the rectangles into bigger ones to avoid broken or missing region. Rectangles are scanned and compared with the others. Two rectangles are merged if they are aligned in the horizontal direction and close to each other. This can be formulated as Eq. (6) to indicate the feasibility of merging.

$$
d=\frac{\text { overlap }_{V}}{\min \left(h_{1}, h_{2}\right)}-3 \times \frac{\text { dist }_{H}}{\sqrt{w_{1} \times w_{2}}}
$$

where ${ }^{\text {overlap }} v$ is the length of the portion that the rectangles overlap vertically, which tells how much they are aligned in the horizontal direction, while ${ }^{d i s_{H}}$ is the distance between the rectangles. $h_{1}, h_{2}, w_{1}, w_{2}$ are the height and width of the rectangles respectively for normalizing the value of $\mathrm{d}$. This is further illustrated in Figure 6.

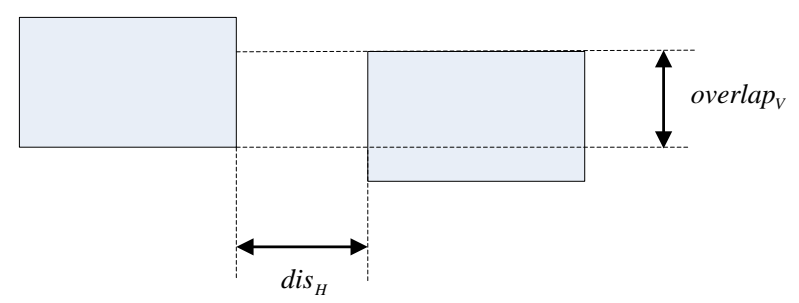

Figure 6: Merging of rectangles

We can see in Eq. (6), a weighting factor of 3 is assigned to magnify the importance of the distance between the two rectangles so as to avoid over-merging. The maximum value of the equation is 1 and in our algorithm, if $\mathrm{d}$ exceeds 0.5 the rectangles are merged. After the merging step, rectangles are further filtered by their size that too small ones are removed.

\subsection{Plate Region Verification}

A verification process is to evaluate the possibility that a candidate region contains any license plate object, and various features can be utilized in the evaluation such as aspect ratio, size. In Macao license plate, there is a country specific feature that a plate must contain the alphabet "M" as the first character. This characteristic is utilized to verify the existence of a plate in our system as well as to determine the region's location and area. An effective technique to identify the " $M$ " in an image is the template matching algorithm. Instead of the conventional way of matching through all sub-regions across the plate region, our algorithm makes heuristic guess on the locations of "M" and matching is performed on those locations only to improve 
computation efficiency. Consider the structure of character " $\mathrm{M}$ " has strong edges at its leftmost and rightmost boundaries, all vertical edges are searched first, by analyzing the vertical projection of the vertical edge map of a candidate region, which is calculated with Eq. (7).

$$
e_{\text {vproj }}^{\prime}(j)=\sum e^{\prime}(i, j)
$$

where $e^{\prime}$ is the sub-region in the vertical edge map $e$ corresponding to a plate region. The set of values is then normalized with their maximum value. For each column $j$, if the projected intensity $e_{v p r o j}^{\prime}$ exceeds a threshold, the column is further scanned and each segment within the column is verified by template matching. An example is given in Figure 7.
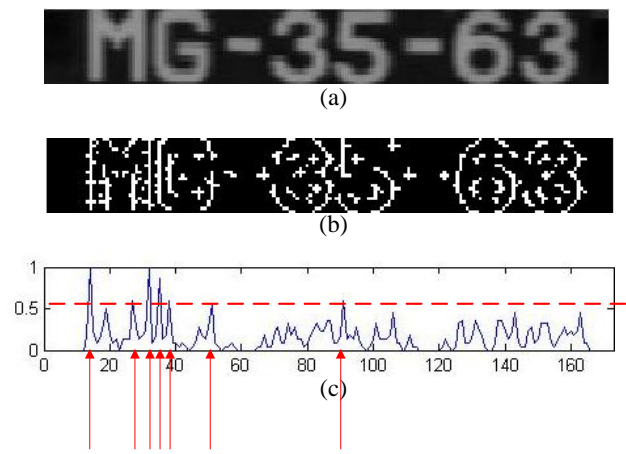

Figure 7: Verification candidate selection. (a) Extracted plate. (b) Edge map. (c) Candidates.

In reality, an edge is usually broken down into a few segments that are scattered across the neighbor columns in the vertical edge map. This is due to the rotation issue of the input image. To cater this issue, an additional step is performed before the matching. For each segment, its surrounding regions are also scanned to ensure broken segments are not missed and they are connected together as a single one. Suppose a segment at column $j$ has a starting point $(i, j)$ with length $l$, we define its four neighbor regions as below:

- Upper-Left - column $j-1$, rows $i-l$ to $i-1$

- Lower-Left - column $j-1$, rows $i+1$ to $i+2 l$

- Upper-Right - column $j+1$, rows $i-l$ to $i-1$

- Lower-Right - column $j+1$, rows $i+1$ to $i+2 l$

The height $h_{R}$ of the sub-region corresponding to the segment is calculated by adding up the lengths of the segment itself and the connected segments in the upper and lower regions, while the width $w_{R}$ is calculated proportionally to $h_{R}$, whose ratio is $5 / 7$. The template image of " $\mathrm{M}$ " is also resized accordingly. Template matching is performed by calculating the crosscorrelation value between the pixel intensities of the sub-region and the template image as formulated in Eq. (8).

$$
\operatorname{cor}=\frac{\sum_{i}^{h_{R}} \sum_{j}^{w_{R}}(R-\bar{R}) \cdot(T-\bar{T})}{\sqrt{\sum_{i}^{h_{R}} \sum_{j}^{w_{R}}(R-\bar{R})^{2} \cdot \sum_{i}^{h_{R}} \sum_{j}^{w_{R}}(T-\bar{T})^{2}}}
$$

where $R$ is the sub-region and $T$ is the template image, $\bar{R}$ and $\bar{T}$ are the mean values respectively. The segment with the maximum correlation value is considered as the location where the first character ("M") resides, and the size of the candidate plate region is also fine-tuned with the information of character's height available.

The result of the extraction algorithm is demonstrated in Figure 8.

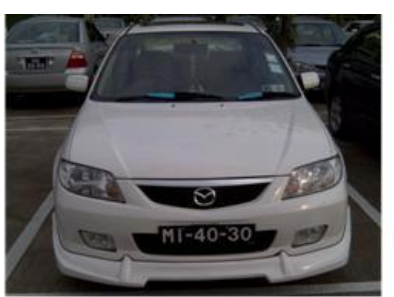

(a)



(b)

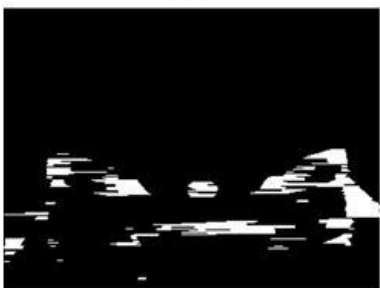

(c)

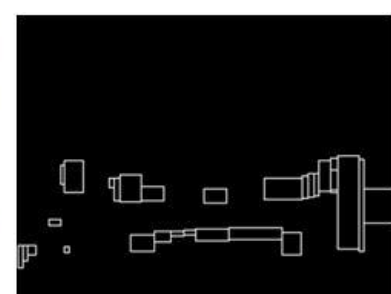

(d)

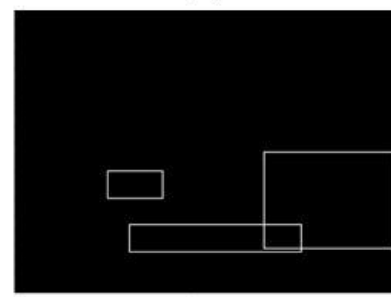

(e)

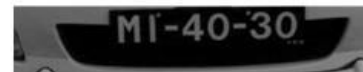

(f)

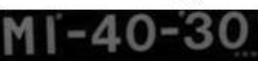

(g)
Figure 8: Extraction result. (a) Original image. (b) Vertical edge map. (c) Edge lines. (d) Line-combined rectangle. (e) Merged rectangle. (f) Identified plate region. (g) Optimized plate region. 


\subsection{Row Separation}

The previous verification algorithm can be applied directly to the license plates with one character row. For two-row type, character " $\mathrm{M}$ " is in the first row and there are other characters in the second row which may also possess strong boundaries and their positions are close to the edges of " $\mathrm{M}$ ". This introduces noise to the projected intensity and affects the accuracy of the algorithm. Thus, the character rows will need to be separated first before applying the verification algorithm.

The candidate regions whose aspect ratio (width-toheight) is larger than 2.5 are considered as one-row type and they are processed with the verification algorithm, otherwise they are considered as two-row type and being processed with row separation algorithm first. The threshold of 2.5 is based on the general aspect ratio of both types of license plates.

Row separation utilizes the nature of intensity transition from peak to valley when it goes from the first row to the background and then to the second row. This feature can be captured with the horizontal projection of the image intensity. Morphological dilation is applied to the license plate image as a pre-processing step to fill up the holes of characters, in order to reduce the influence on intensity changes caused by character structure itself. The structuring element is a horizontal line with its length equals to one-fifth of the character width. Suppose $f_{\text {dil }}$ is the dilated region, the horizontal projection is calculated with Eq. (9).

$$
f_{\text {dil hproj }}(i)=\sum_{j} f_{d i l}(i, j)
$$

The set of values is then normalized with their maximum value of $f_{d i l}$ hmpri . When the projection is scanned from top to down, there is a drop in the horizontal intensity when it transits between the two character-rows and the separation area. Ideally, a global threshold can be used to identify this intensity drop and subsequently its location of separation. In reality, this threshold needs to be flexible depending on the image capturing condition and the extraction result. For example, in Figure 9(b), a slanted license plate still gives a strong intensity at its separation area due to the influence of the character-rows. In Figure 9(c), the extracted plate image contains part of the vehicle body and thus the overall intensity at the license plate area is weakened.
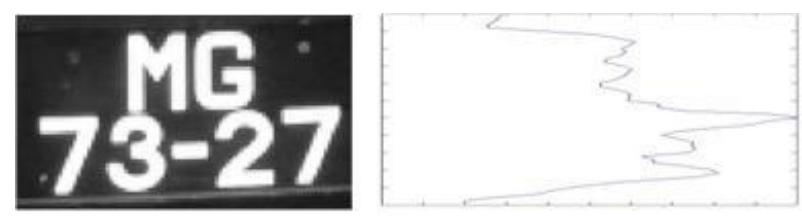

(a)


(b)

Figure 9: Examples of horizontal projection result of two-row license plates. (a) Slanted case. (b) Undesired region is included.

Instead of using a fixed threshold, a searching mechanism is proposed in our system to identify the separation location by comparing the difference between the local minima and maxima of the horizontal projected intensity. $L_{\min }$ is defined as a set of local minima of $f_{\text {dil hrproj }}$ and their corresponding position. It is expressed as Eq. (10).

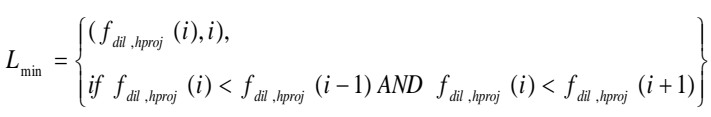

$f_{\text {dit h hroj }}(i)$ at row $i$ is considered as a local minimum if both of its neighbor rows have a bigger intensity value than itself. Similar to (10), $L_{\max }$ is defined as a set of local maxima of $f_{\text {dil h, hroj }}$ and their corresponding position. It is expressed as Eq. (11).

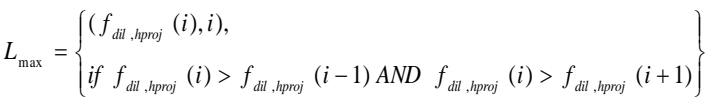

The intensity transition is a rapid change in the values of $f_{\text {dil hroroj }}$ from a local minimum to a local maximum within a few rows in the image. In the implementation, For each local minimum $L_{\min }(p)$, we calculate its intensity rate-of-change as Eq. (12):

$$
r(p)=\frac{f_{\text {dil }, \text { hprj }}(p)-f_{\text {dil }, \text { hprj }}(q)}{p-q}
$$

where $\left(f_{\text {dil hhroj }}(q), q\right)$ must be a local maximum in $L_{\max }$ and $q$ is the nearest row after $p$ in the image. The separation area has a relatively high rate-of-change value due to the big difference between the values of its 
local minimum $f_{\text {dil }, \text { hproj }}(p)$ and maximum $f_{\text {dil ,hproj }}(q)$ while the distance between $p$ and $q$ is small.

Any row with its rate-of-change values exceeds a threshold $t h_{R O C}$ is considered as a candidate of separation location. The upper half of the license plate is then further examined with the plate region verification algorithm. The row with the highest score is selected as the final separation line and the plate region is adjusted accordingly. ${ }^{t h}{ }_{R O C}$ is calculated by the Eq. (13).

$$
t h_{R O C}=\frac{0.2}{t h_{L_{-} D I S T}}=\frac{0.2}{0.01 \times w_{I M G}}=\frac{20}{w_{I M G}}
$$

The reason behind is in the worst case scenario, the minimum difference between a local minimum and maximum is 0.2 throughout the experiment. The threshold is calculated by dividing it with the maximum possible distance between the two character rows, which is $t h_{L N}$ DIST as calculated with Eq. (13).

The result of row separation for two-row plates is given in Figure 10.

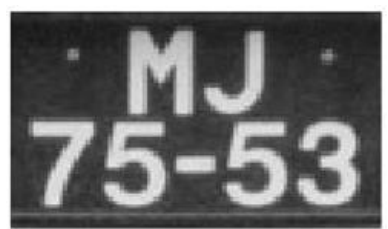

(a)

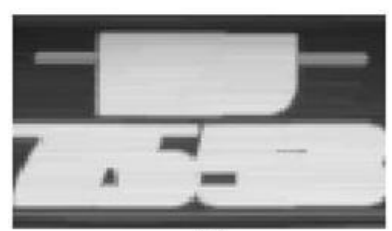

(b)

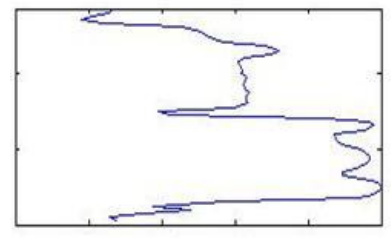

(c)

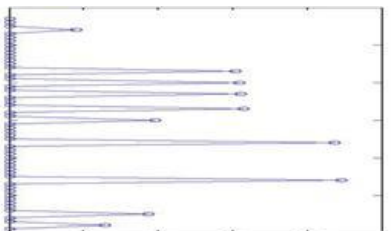

(d)

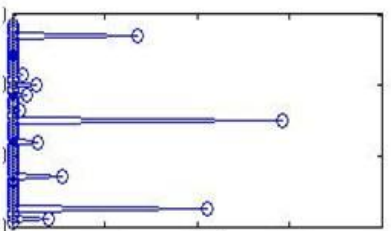

(e)

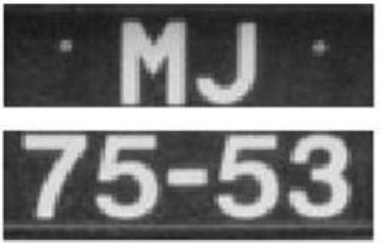

(f)
Figure 10: Row Separation. (a) Original image. (b) Dilated image. (c) Horizontal projection. (d) Local minima graph. (e) Rate-of-change graph. (f) Separation result.

\section{Character Segmentation}

In Macao license plate, various character fonts are available whose character size and spacing are different from each other, thus no single rule can be applied to extract the character objects. Characters in Macao license plate are machine-printed and separable in columns. Base on the plate image output from the extraction stage, we analyze its pattern of intensity change with vertical projection in order to identify the background areas between each character (we name it as character separators thereafter in this paper) which are used to segment the plate image in a dynamic manner.

Before the analysis, pre-processing with morphological erosion is applied for better performance. In many cases, characters are close to each other from the captured image, for example, angle between camera and vehicle, or old character fonts which have negative effects on the intensity analysis. Erosion can shrink the boundaries of characters and increase the space between the characters. The structuring element in this step is a square with its length equals to one-fifth of the character width. Another pre-processing step with morphological dilation is also applied to fill up the holes of characters, which is similar to the row separation process. The structuring element is a vertical line with its length equals to twice of the character width.

The vertical projection and its local minima set are obtained with the formulas similar to (7) and (9) respectively. Each local minimum is a separator and a threshold $t_{c s}$ is then selected for the local minima set in order to isolate the character-separator set from the non-separator set. The threshold is calculated with Eq (14):

$$
t h_{C S}=\mu\left(L_{\min }\right)+\frac{1}{3} \sigma\left(L_{\min }\right)
$$

where $\mu$ is the mean and $\sigma$ is the standard derivation of the local minima value. The license plate is then segmented with the remaining separators. An example is given in Figure 11 on the segmentation process.

The resulting segments are then binarized and trimmed to remove the redundant space and undesirable elements (e.g. blot) for recognition process. Furthermore, a trimmed segment is ignored if its aspect ratio does not match the ratio of normal characters such that non-character-like objects (e.g. the hyphens between plate numbers) are filtered away. At the end, a set of character objects are output to the character recognition process. 
(a)



(b)



(c)

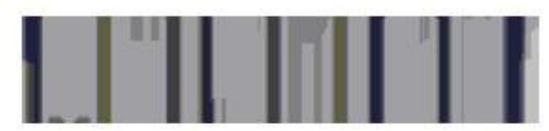

(d)

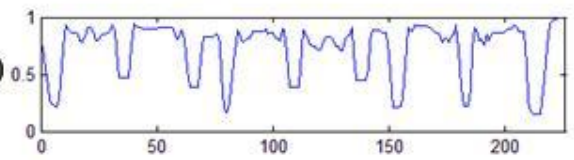

(e)

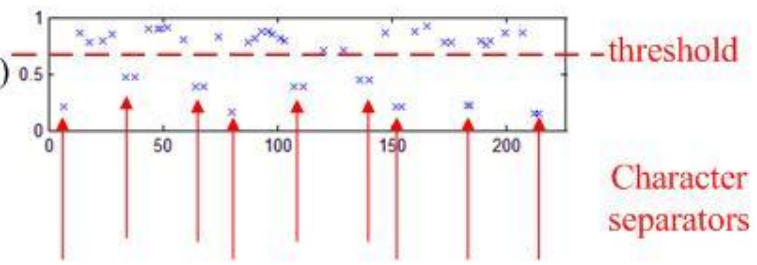

(f)

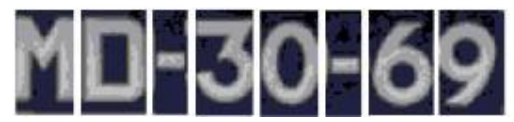

Figure 11: Character Segmentation. (a) Extracted plate image. (b) Eroded image. (c) Dilated image. (d) Vertical projection. (e) Local minima graph and threshold selection. (f) Resulting character segments.

\section{Character Recognition}

This process is accomplished by template matching. It is straightforward and reliable in the Macao license plate domain since it contains only English alphabets and Arabic numerals with a finite number of font types. Also the computational effort is relatively low compared to neural network method which is another majority approach.

Before matching takes place, the size of each character segment is normalized into a unified one of $70 \times 50$ pixels, which is the identical to the size of template images. This is achieved by the bilinear interpolation method. For each of the resized character segments, it is matched with the template images by cross-correlation. A template is considered as a successful match if it fulfills the below conditions:

(1) The correlation value exceeds 0.75 (maximum is 1).

(2) The correlation value is the highest among the other template images.

If no template fulfills these conditions, the algorithm continues with the next character segment until all segments are checked. Base on the prior knowledge of the alphabet and numeral sequence of Macao license plate, the template images being matched are refreshed with either alphabets or numerals accordingly to reduce the number of matching cycles. This way the potential ambiguity between some of the alphabets and numerals can also be mitigated (e.g. "0" and "O", "1" and "I").

Template matching with merely pixel intensity is sensitive to noise and the recognition rate will be degraded if the characters are not in its normal condition (e.g. slanted in shape or undesired objects are included in the segment). This can be improved by introducing additional features for matching. In our algorithm, vertical and horizontal projections of the character segment intensity are used as the features of matching since they contain information of character structure but less rigid than exact pixels. The score of recognition is formulated as Eq. (15):

$$
\text { score }=0.6 \times \text { cor }_{I}+0.2 \times \text { cor }_{V}+0.2 \times \text { cor }_{H}
$$

where $\mathrm{cor}_{I}, \mathrm{cor}_{V}, \mathrm{cor}_{H}$ are the correlation values of the character segment and the template image, corresponding to the pixel intensity, vertical projection, and horizontal projection respectively. Each of them are multiplied with a weight and thus giving more importance to some of the features.

The result of the character recognition process will be the license plate number of the vehicle in the captured image.

\section{Experimental Results and Discussion}

Matlab R2006b is the platform we used to implement this LPR system. In this study, a CPU with Intel Core 2 Duo T7100 1.8GHz and 2GB memory are employed for the test. The test includes 100 images of 24-bit true color with size $640 \times 480$, which are converted into grayscale images. They are taken under various conditions: (1) Normal license plates in one-row / two-row types with various font types. (2) Slanted license plates. (3) Multiple license plates exist in an image. (4) Vehicle and background have high illumination contrast. (5) Vehicle and license plate have similar pattern.

The extraction and recognition performance is summarized in Table 1. It is also compared with another work which aims at solving Macao LPR problems. 
Table 1. Performance result with comparison against other Macao LPR works

\begin{tabular}{|l|c|c|c|}
\hline \multicolumn{1}{|c|}{ Criterion } & $\begin{array}{c}\text { Proposed } \\
\text { LPR }\end{array}$ & $\begin{array}{c}\text { Work of } \\
{[13]}\end{array}$ & $\begin{array}{c}\text { Work of } \\
{[4]}\end{array}$ \\
\hline Extraction Rate & $100 \%$ & $95 \%$ & $93.2 \%$ \\
\hline Recognition Rate & $95 \%$ & $\mathrm{n} / \mathrm{a}$ & $88 \%$ \\
\hline $\begin{array}{l}\text { Overall } \\
\text { Recognition Rate }\end{array}$ & $95 \%$ & $\mathrm{n} / \mathrm{a}$ & $82.3 \%$ \\
\hline
\end{tabular}

In [13], an edge based license plate extraction scheme was implemented. Two features were employed to verify a candidate region: (1) aspect ratio, and (2) proportion of pixels in black color within the region. Moreover, an input image would be scaled into three different levels and gone through the same extraction process so that the results would be fused together to boost the accuracy. This algorithm was able to achieve a high extraction rate of $95 \%$, nevertheless it is suspicious that the processing time could be degraded due to redundant cycles of computation. Our algorithm has followed the same approach of edge processing, however, with the revised region merging scheme and verification method, we successfully improve the extraction rate by $5 \%$. Furthermore, our verification method by searching for the character " $\mathrm{M}$ " is a one-off process which will be faster than the method in [13]. Character segmentation and recognition processes were not discussed in [13] and thus comparison with recognition rate is not applicable.

In [4], a morphology based license plate extraction algorithm was implemented and our edge-based extraction algorithm has a better extraction rate by $6.8 \%$. For character segmentation and recognition processes, they utilized the projection method and template matching method respectively. Their recognition reached $88 \%$ merely which is relatively unsatisfactory. Our algorithms have utilized the same techniques with additional pre-processing steps to resolve several obstacles such as slanted plates, and successfully achieve a higher recognition rate of $95 \%$. The overall performance gain is $12.7 \%$ by comparing the overall recognition rate.

Specific examples in handling exceptional scenarios are given in Figure 12, 13, 14 and 15.



Original Image

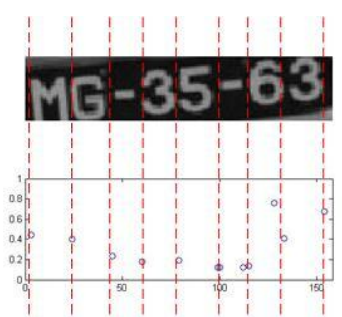

Extraction and Segmentation Result
Recognition Result: M G 3563

Figure 12: Experimental result with slanted license plate

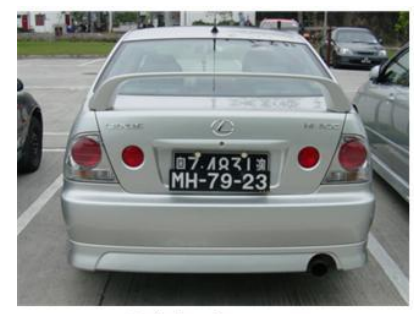

Original Image

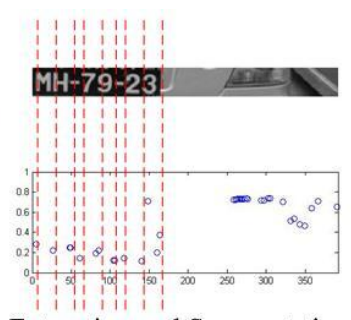

Extraction and Segmentation Result
Recognition Result: M H 7923

Figure 13: Experimental result with multiple license plates

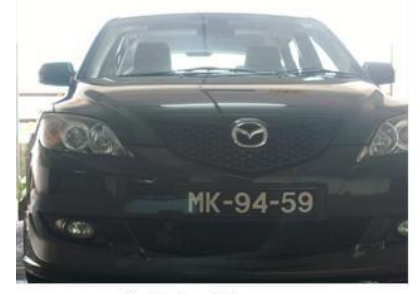

Original Image

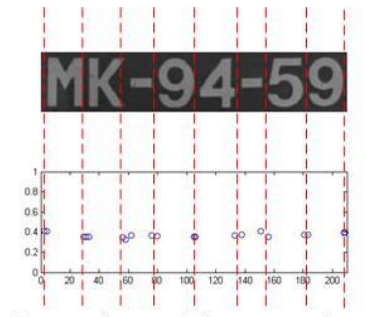

Extraction and Segmentation Result
Recognition Result: M K 9459

Figure 14: Experimental result with high illumination contrast between background and license plate 


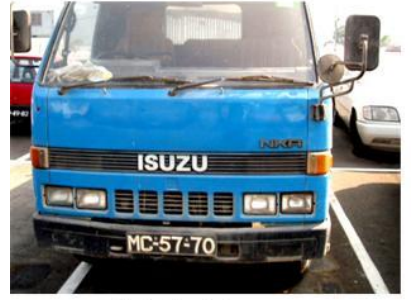

Original Image



Extraction and Segmentation Result
Recognition Result: M C 5770

Figure 15: Experimental result with plate-like pattern.

\section{Conclusion}

In this paper, the Sobel edge detection has been employed to retrieve the vertical edges of the input image. The edges are then combined into candidate regions with the composition algorithm, by combining the edge points into horizontal lines and eventually rectangles. Each region is further verified on its validity by determining the existence of the character " $M$ ". It is a Macao country specific feature that license plate number must contain the character " $M$ " as the first character. Our system has also catered both one-row and two-row types of plates, in which, two-row type is processed with the row separation algorithm by horizontal projection analysis. The proposed scheme is able to achieve a $100 \%$ extraction rate with our testing samples. Its robustness is proven.

In the character segmentation stage, character separators are identified based on vertical projection analysis of the extracted plate image's pixel intensity. A pair of separators will form a character segment. Various pre and post processing steps such as morphological erosion and trimming are exploited to tackle the issues encountered in segmentation. In the last stage, the character segments are resized and recognized by template matching which includes three features: exact pixel intensity, as well as its vertical projection value and horizontal projection value. This reduces the drawback of the template matching method that it is very sensitive to noise. Experimental result gives a high recognition rate of $95 \%$ which is satisfactory.

Our work has It also achieved a major improvement than another existing works which is designed for solving Macao LPR problems.

\section{Acknowledgements}

The authors would like to thank the referees for their valuable comments. This work was supported in part by the Research Committee of the University of Macau.

\section{References}

[1] Mei Yu; Yong Deak Kim, "An approach to Korean license plate recognition based on vertical edge matching," Systems, Man, and Cybernetics, 2000 IEEE International Conference on , vol.4, no., pp.2975-2980 vol.4, 2000

[2] Bai Hongliang; Liu Changping, "A hybrid license plate extraction method based on edge statistics and morphology," Pattern Recognition, 2004. ICPR 2004. Proceedings of the 17th International Conference on , vol.2, no., pp. 831-834 Vol.2, 23-26 Aug. 2004

[3] Ahmed, M.J.; Sarfraz, M.; Zidouri, A.; Al-Khatib, W.G., "License plate recognition system," Electronics, Circuits and Systems, 2003. ICECS 2003. Proceedings of the 2003 10th IEEE International Conference on , vol.2, no., pp. 898-901 Vol.2, 14-17 Dec. 2003

[4] Cheokman Wu; Lei Chan On; Chan Hon Weng; Tong Sio Kuan; Kengchung Ng, "A Macao license plate recognition system," Machine Learning and Cybernetics, 2005. Proceedings of 2005 International Conference on , vol.7, no., pp.4506-4510 Vol. 7, 18-21 Aug. 2005

[5] Shyang-Lih Chang; Li-Shien Chen; Yun-Chung Chung; Sei-Wan Chen, "Automatic license plate recognition," Intelligent Transportation Systems, IEEE Transactions on , vol.5, no.1, pp. 42-53, March 2004

[6] Wu Wei; Yuzhi Li; Mingjun Wang; Zhongxiang Huang, "Research on number-plate recognition based on neural networks," Neural Networks for Signal Processing XI, 2001. Proceedings of the 2001 IEEE Signal Processing Society Workshop , vol., no., pp.529-538, 2001

[7] Tsang-Hong Wang; Feng-Chou Ni; Keh-Tsong Li; YonPing Chen, "Robust license plate recognition based on dynamic projection warping," Networking, Sensing and Control, 2004 IEEE International Conference on , vol.2, no., pp. 784-788 Vol.2, 2004

[8] Yungang Zhang; Changshui Zhang, "A new algorithm for character segmentation of license plate," Intelligent Vehicles Symposium, 2003. Proceedings. IEEE, vol., no., pp. 106-109, 9-11 June 2003

[9] Pan Xiang; Ye Xiuzi; Zhang Sanyuan, "A hybrid method for robust car plate character recognition," Systems, Man and Cybernetics, 2004 IEEE International Conference on , vol.5, no., pp.4733-4737 vol.5, 0-0 0

[10] A. Broumandnia and M. Fathi, "Application of pattern recognition for farsi license plate recognition", Intl. J. Graphics, Vis. Image Process., vol. V2, pp. 25, Jan. 2005.

[11] Naito, T.; Tsukada, T.; Yamada, K.; Kozuka, K.; Yamamoto, S., "Robust license-plate recognition method for passing vehicles under outside environment," 
Vehicular Technology, IEEE Transactions on , vol.49, no.6, pp.2309-2319, Nov 2000

[12] Sirithinaphong, T.; Chamnongthai, K., "Extraction of car license plate using motor vehicle regulation and character pattern recognition," Circuits and Systems, 1998. IEEE APCCAS 1998. The 1998 IEEE Asia-Pacific Conference on , vol., no., pp.559-562, 24-27 Nov 1998

[13] Wen-Li Yao; Chi-Man Pun, "A hybrid Macau license plate localization scheme based on vertical edge analysis," Machine Learning and Cybernetics, 2009 International Conference on , vol.3, no., pp.1544-1549, 12-15 July 2009

[14] Macao Rules of Transportations, available at: http://bo.io.gov.mo/bo/i/93/17/codestcn/declei17an.asp, accessed Dec-2009 